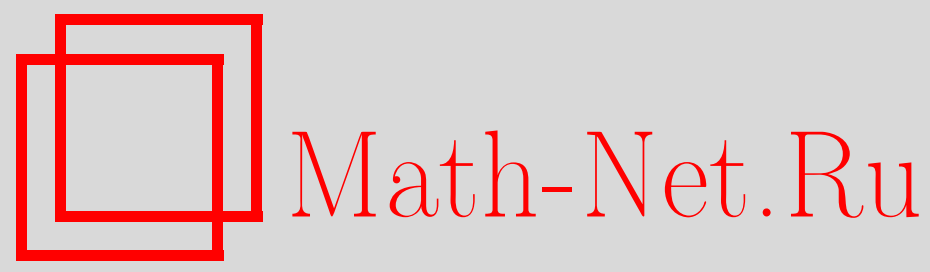

С. В. Зелик, Ограниченность решений нелинейной эллиптической системы в цилиндрической области, Матем. заметки, 1997, том 61, выпуск 3, 447-450

DOI: https://doi.org/10.4213/mzm1520

Использование Общероссийского математического портала Math-Net.Ru подразумевает, что вы прочитали и согласны с пользовательским соглашением http://www . mathnet.ru/rus/agreement

Параметры загрузки:

IP : 54.237 .59 .107

26 апреля 2023 г., 15:30:51

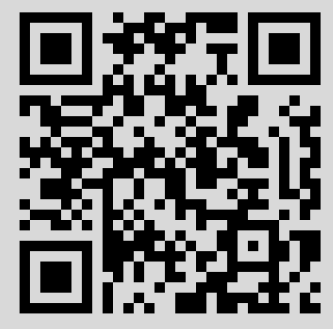




\section{ОГРАНИЧЕННОСТЬ РЕШЕНИЙ НЕЛИНЕЙНОЙ ЭЛЛИПТИЧЕСКОЙ СИСТЕМЫ В ЦИЛИНДРИЧЕСКОЙ ОБЛАСТИ}

\section{С. В. Зелик}

В полуцилиндре $\Omega_{+}=\mathbb{R}_{+} \times \omega$, где $\omega-$ ограниченная область в $\mathbb{R}^{n}$ с достаточно гладкой границей, рассматривается следующая эллиптическая система:

$$
\begin{aligned}
& a\left(\partial_{t}^{2} u+\Delta u\right)+\gamma \partial_{t} u-f(u, t)=g(t), \\
& \left.u\right|_{t=0}=u_{0},\left.\quad u\right|_{\partial \omega}=0 .
\end{aligned}
$$

Здесь $u=u(t, x)=\left(u_{1}, \ldots, u_{k}\right), g=g(t, x), f(u, t)$ - векторные функции, $(t, x) \in \Omega_{+}$, $\Delta$ - оператор Лапласа по переменной $x=\left(x_{1}, \ldots, x_{n}\right), \gamma$ и $a$ - постоянные матрищы $\left(\gamma, a \in L\left(\mathbb{R}^{k}, \mathbb{R}^{k}\right)\right)$, причем $a=a^{*}>0$. Предполагается, что $g$ принадлежит пространству $\left[L_{p}^{\text {loc }}\left(\mathbb{R}_{+}, L_{p}(\omega)\right)\right]^{k}$ для некоторого $p>n+1$ и имеет конечную норму

$$
|g|_{a}=\sup _{t \in \mathbb{R}_{+}}\left\|g, \Omega_{t}\right\|_{L_{p}}<\infty, \quad \text { где } \Omega_{t}=[t, t+1] \times \omega .
$$

Нелинейная функция $f$ удовлетворяет следующим условиям:

$$
\begin{aligned}
& f \in C\left(\mathbb{R}^{k} \times \mathbb{R}_{+}, \mathbb{R}^{k}\right), \\
& f(u, t) . u \geqslant-C_{1}+C_{2}|u|^{2+\varepsilon}, \quad \varepsilon>0, \quad C_{2}>0, \\
& |f(u, t)| \leqslant Q(|u|), \quad \text { где } Q: \mathbb{R}_{+} \rightarrow \mathbb{R}_{+}-\text {монотонная функция. }
\end{aligned}
$$

Здесь и далее, через $u . v$ обозначается скалярное произведение в $\mathbb{R}^{k}$. Начальное условие $u_{0}$ предполагается принадлежащим пространству

(см. [1]).

$$
V_{0}=\left[H_{2-1 / p, p}(\omega) \cap H_{1, p}^{0}(\omega)\right]^{k} \quad\left(u_{0} \in V_{0}\right)
$$

Под решением задачи (1) будем понимать функцию $u$, принадлежащую пространству $\left[H_{2, p}\left(\Omega_{t}\right)\right]^{k} \forall t>0$, где $\Omega_{t}=[t, t+1] \times \omega$, и удовлетворяющую $(1)$ в смысле равенства в $\left[L_{p}^{\text {loc }}\left(\Omega_{+}\right)\right]^{k}$. (Здесь и далее, через $H_{l, p}$ обозначается соболевское пространство функций, обобщенные производные которых до порядка $l$ включительно принадлежат пространству $\left.L_{p}[2].\right)$ Множество всех решений задачи (1) в указанном вьше смысле обозначим символом $\mathscr{V}\left(u_{0}\right)$.

Под ограниченным решением задачи (1) понимается решение $u$, дополнительно удовлетворяющее следующему условию:

$$
\|u\|_{a}=\sup _{t \in \mathbb{R}_{+}}\left\|u, \Omega_{t}\right\|_{H_{2, p}}<\infty .
$$

Множество всех ограниченных решений задачи (1) обозначим символом $\mathscr{V}_{a}\left(u_{0}\right)$.

Ограниченные решения задачи (1) при различных условиях на нелинейную функцию $f$ изучались многими авторами [3]-[5].

В работе [5] доказано, что при указанных выше условиях множество $\mathscr{V}_{a}\left(u_{0}\right)$ не пусто, и получена следующая оценка:

$$
\left\|u, \Omega_{T}\right\|_{2, p} \leqslant R\left(\left\|u_{0}\right\|_{V_{0}}\right) e^{-\alpha T}+R\left(|g|_{a}\right) .
$$

Здесь $\alpha>0$ и $R$-некоторая монотонная функция, не зависящая от $u_{0}$.

Основной результат настоящей работы сформулируем в виде следующей теоремы.

Работа выполнена при частичной поддержке Российского фонда фундаментальных исследований, грант № 96-01-00354.

(C) С.В. ЗЕлик 
ТЕорема 1. Пусть выполнены перечисленные выше условия. Тогда $\mathscr{V}\left(u_{0}\right)=$ $\mathscr{V}_{a}\left(u_{0}\right)$ и справедлива следующая оченка, усиливающая оченку (3):

$$
\left\|u, \Omega_{T}\right\|_{2, p} \leqslant R\left(\left\|u_{0}\right\|_{V_{0}}\right) \chi\left(T_{0}-T\right)+R\left(|g|_{a}\right) .
$$

Здесь $\chi(z)$ - функиия Хевисайда, равная нулю при $z \leqslant 0$ и единиче при $z>0, a$ $T_{0}=T_{0}\left(f,|g|_{a}\right)>0$ - некоторое число, не зависящее от $u_{0}$.

Для доказательства теоремы 1 нам понадобится ряд вспомогательных утверждений, имеющих и некоторый самостоятельный интерес.

Лемма 1. Пусть $u_{0} \in \mathscr{V}\left(u_{0}\right)$ и $y(t)=\left(|u|^{p}, 1\right)$, где $(u, v)$ - скалярное произведение в пространстве $L_{2}(\omega),|u|=[\text { аи. } u]^{1 / 2}$. Тогда $y^{\prime \prime}(t) \in L_{1}^{\operatorname{loc}}\left(\mathbb{R}_{+}\right)$и удовлетворяет неравенству

$$
y^{\prime \prime}(t)-\beta^{2} y(t)|y(t)|^{\alpha} \geqslant h(t)
$$

при некоторых $\alpha>0, \beta>0 u h(t)=-C\left(1+\|g(t)\|_{0, p}^{p}\right)$.

ДокАЗАтЕльство. Умножая уравнение (1) скалярно на $u|u|^{p-2}$ в $\left[L_{2}(\omega)\right]^{k}$, после несложных преобразований получим

$$
\begin{aligned}
& \frac{1}{p} \partial_{t}^{2}\left(|u|^{p}, 1\right)-\frac{4(p-2)}{p^{2}}\left(\left\|\partial_{t}|u|^{p / 2}\right\|_{0,2}^{2}+\left\|\nabla|u|^{p / 2}\right\|_{0,2}^{2}\right) \\
& \quad=-\left(\gamma \partial_{t} u, u|u|^{p-2}\right)+\left(g, u|u|^{p-2}\right)+\left(f(u), u|u|^{p-2}\right)+\left(\left|\partial_{t} u\right|^{2}+|\nabla u|^{2},|u|^{p-2}\right) .
\end{aligned}
$$

Оценим снизу правую часть неравенства (6) при помощи неравенства Гёльдера и условия (2). Получим

$$
\partial_{t}^{2}\left(|u(t)|^{p}, 1\right)-C\left(|u(t)|^{p+\varepsilon}, 1\right) \geqslant h(t) .
$$

Оценим второе слагаемое формулы (7) с помощью неравенства Иенсена

$$
\left(|u(t)|^{p+\varepsilon}, 1\right)=\int_{\omega}|u(t)|^{p+\varepsilon} d x=\int_{\omega}\left[|u(t)|^{p}\right]^{1+\varepsilon / p} d x \geqslant C(\omega)\left(\int_{\omega}|u(t)|^{p} d x\right)^{1+\varepsilon / p} .
$$

Подставив оценку (8) в неравенство (7), получим оценку (5).

Лемма 2. Пусть $y(t) \geqslant 0, y^{\prime \prime} \in L_{1}^{\text {loc }}\left(\mathbb{R}_{+}\right)$и удовлетворяет неравенству (5). Тогда

$$
y(t) \leqslant C\left(y(0) \chi\left(t_{0}-t\right)+1+\sup _{T \geqslant 0} \int_{T}^{T+1}|h(t)| d t\right) .
$$

Здесь $C$ и $t_{0}$ не зависят от $y$.

Доказательство этого утверждения носит технический характер и потому здесь не приводится.

СлеДСТвИЕ. Пусть $u \in \mathscr{V}\left(u_{0}\right)$. Тогда справедлива оценка

$$
\|u(t)\|_{0, p} \leqslant C_{1}\left(\chi\left(t_{0}-t\right)\left\|u_{0}\right\|_{V_{0}}+1+|g|_{a}+1\right), \quad C_{1}>0 .
$$


Лемма 3. Пусть $u \in \mathscr{V}\left(u_{0}\right)$. Тогда при $T \geqslant 1$ справедлива оценка

$$
\left\|u, \Omega_{T}\right\|_{0, \infty} \leqslant C\left(1+\left\|u, \Omega_{T-1, T+2}\right\|_{0, p}+|g|_{a}\right) .
$$

Здесъ $\Omega_{T-1, T+2}=[T-1, T+2] \times \omega$.

ДоказАтельство. Умножим уравнение (1) скалярно на $u|u|^{q-2}$ в $\left[L_{2}(\omega)\right]^{k}(q \geqslant 2)$. Рассуждая так же, как и при вьводе формул (6) и (7), получим

$$
\left\|\partial_{t}|u|^{q / 2}\right\|_{0,2}^{2}+\left\|\nabla|u|^{q / 2}\right\|_{0,2}^{2} \leqslant C q\left(\partial_{t}^{2}\left(|u|^{q}, 1\right)+\left(g, u|u|^{q-2}\right)+\left(|u|^{p}, 1\right)\right) .
$$

Рассмотрим теперь семейство областей $G^{m}=\left[T-2^{-m}, T+1+2^{-m}\right], m \geqslant 0$, $\Omega^{m}=G^{m} \times \omega$ и семейство срезающих функций $\varphi_{m}(t) \in C_{0}^{\infty}(\mathbb{R})$ таких, что $\varphi_{m}(t) \equiv 1$ при $t \in G^{m+1}$ и $\varphi_{m}(t) \equiv 0$ при $t \notin G^{m}$. Очевидно, что семейство $\varphi_{m}$ может быть выбрано так, чтобы $\left|\varphi_{m}^{\prime \prime}(t)\right| \leqslant C 2^{2 m}$, где $C$ не зависит от $m$. Умножив соотношение (10) на $\varphi_{m}(t) \geqslant 0$, проинтегрировав по $t$ и использовав элементарную формулу

$$
\varphi_{m} \partial_{t}^{2} F=\partial_{t}\left(\varphi_{m} \partial_{t} F\right)-\partial_{t}\left(\varphi_{m}^{\prime} F\right)+\varphi_{m}^{\prime \prime} F, \quad F=\left(|u|^{q}, 1\right),
$$

получим следующую оценку:

$$
\left\||u|^{q / 2}, \Omega^{m+1}\right\|_{1,2}^{2} \leqslant C_{1} q 2^{2 m}\left(1+\left\|u, \Omega^{m}\right\|_{0, q}^{q}+\int_{\Omega^{m}} g . u|u|^{q-2} d x d t\right) .
$$

Согласно теореме вложения С. Л. Соболева (см. [1])

$$
\left\|u, \Omega^{m+1}\right\|_{q(n+1) /(n-1)}^{q} \leqslant C\left\||u|^{q / 2}, \Omega^{m+1}\right\|_{1,2}^{2} .
$$

Оценивая последний интеграл в формуле (11) при помощи неравенства Гёльдера, получим

$$
\int_{\Omega^{m}} g \cdot u|u|^{q-2} d x d t \leqslant|g|_{a}^{q}+\left\|u, \Omega^{m}\right\|_{0, q(n+1) / n}^{q} .
$$

Подставив оценки (12) и (13) в неравенство (11), будем иметь

$$
\left\|u, \Omega^{m+1}\right\|_{q(n+1) /(n-1)}^{q} \leqslant C_{2} q 2^{2 m}\left(\left\|u, \Omega^{m}\right\|_{0, q(n+1) / n}^{q}+\left(1+|g|_{a}\right)^{q}\right) .
$$

Введем обозначение

$$
W_{l}^{m}(u)=\max \left\{\left\|u, \Omega^{m}\right\|_{0, l},\left(1+|g|_{a}\right)\right\} .
$$

С учетом (15) после извлечения из (14) корня степени $q$, получим

$$
W_{l(\delta+1)}^{m+1}(u) \leqslant\left[C_{3} l 2^{2 m}\right]^{(n+1) /(l n)} W_{l}^{m}(u) .
$$

Здесь $l=n q /(n+1), \delta=1(n-1)$.

Определим последовательность $l_{m}=p(\delta+1)^{m}$. Тогда из (16) следует оценка

$$
W_{l_{m+1}}^{m+1}(u) \leqslant A_{m} W_{l_{m}}^{m}(u), \quad A_{m}=\left[C_{3} l_{m} 2^{2 m}\right]^{(1+1 / n) / l_{m}} .
$$

Итерируя неравенство (17) $m$ раз, получим

$$
\left\|u, \Omega^{m}\right\|_{0, l_{m}} \leqslant P_{m}\left(1+|g|_{a}+\left\|u, \Omega^{0}\right\|_{0, p}\right), \quad P_{m}=A_{0} \times \cdots \times A_{m} .
$$

Нетрудно проверить, что

$$
\lim _{m \rightarrow \infty} P_{m}=C<\infty
$$

следовательно,

$$
\left\|u, \Omega_{T}\right\|_{0, l_{m}} \leqslant C\left(1+|g|_{a}+\left\|u, \Omega^{0}\right\|_{0, p}\right) .
$$

Так как $H_{2, p}\left(\Omega_{T}\right) \subset C\left(\Omega_{T}\right)$ согласно теореме вложения, то, переходя к пределу в формуле (18), получаем оценку (9). 
СЛЕДСТВИЕ. Пусть $u \in \mathcal{V}\left(u_{0}\right)$. Тогда справедлива оценка

$$
\left\|u, \Omega_{T}\right\|_{0, \infty} \leqslant C\left(\chi\left(t_{0}-T\right)\left\|u_{0}\right\|_{V_{0}}+1+|g|_{a}\right) .
$$

ЛЕмма 4. Пусть $u \in \mathscr{V}\left(u_{0}\right)$. Тогда справедлива оценка

$$
\left\|u, \Omega_{T}\right\|_{2, p} \leqslant R\left(\left\|u, \Omega_{T-1, T+2}\right\|_{0, \infty}\right)+C|g|_{a} .
$$

Здесь $R: \mathbb{R}_{+} \rightarrow \mathbb{R}_{+}-$некоторая монотонная функиия.

Доказательство этой леммы приведено в работе [5].

ДокАЗАТЕЛЬСТво теОРемЫ 1 . Пусть $u \in \mathscr{V}\left(u_{0}\right)$. Тогда, подставив оценку (19) в формулу (20), получаем оценку (4). Следовательно, $\mathscr{V}\left(u_{0}\right) \subset \mathscr{V}_{a}\left(u_{0}\right)$. Обратное включение очевидно. Теорема 1 доказана.

Приведем в заключение аналог оценки (4) для случая эллиптической системы в ограниченном цилиндре.

Tеорема 2. Пусть $\Omega=[0, M] \times \omega u и \in\left[H_{2, p}(\Omega)\right]^{k}-$ решение следующей задачи:

$$
\begin{aligned}
& a\left(\partial_{t}^{2} u+\Delta u\right)+\gamma \partial_{t} u-f(u, t)=g(t), \\
& \left.u\right|_{t=0}=u_{0},\left.\quad u\right|_{\partial \omega}=0,\left.\quad u\right|_{t=M}=u_{M} .
\end{aligned}
$$

Тогда справедлива следующая оценка:

$$
\left\|u, \Omega_{T}\right\|_{2, p} \leqslant R\left(\left\|u_{0}\right\|_{V_{0}}\right) \chi\left(T_{0}-T\right)+R\left(\left\|u_{M}\right\|_{V_{0}}\right) \chi\left(T-M+T_{0}\right)+R\left(|g|_{a}\right) .
$$

Институт проблем передачи информации РАН

Поступило 03.12 .96

\section{СПИСОК ЦИТИРОВАННОЙ ЛИТЕРАТУРЫ}

1. Трибель Х. Теория интерполяции, функциональные пространства, дифференциальные операторы. М.: Мир, 1980. 2. Соболев С. Л. Некоторые приложения функционального анализа в математической физике. М.: Наука, 1988. 3. Calsina A., Mora X., Sola-Morales J. // J. Differential Equations. 1993. V. 102. P. 244-304. 4. Бабин А. В. // Изв. РАН. Сер. матем. 1994. Т. 58. №2. С. 3-18. 5. Вишик М. И., Зелик С. В. // Матем. сб. 1996. Т. 187. № 12. С. 21-56. 